\title{
Supporting smartphone-based behavioral activation
}

A simulation study

Bardram, Jakob Eyvind ; Rohani, Darius A.; Tuxen, Nanna; Faurholt-Jepsen, Maria; Kessing, Lars V.

Published in:

UbiComp/ISWC 2017 - Adjunct Proceedings of the 2017 ACM International Joint Conference on Pervasive and Ubiquitous Computing and Proceedings of the 2017 ACM International Symposium on Wearable Computers

Link to article, DOI:

$10.1145 / 3123024.3125617$

Publication date:

2017

Document Version

Peer reviewed version

Link back to DTU Orbit

Citation (APA):

Bardram, J. E., Rohani, D. A., Tuxen, N., Faurholt-Jepsen, M., \& Kessing, L. V. (2017). Supporting smartphonebased behavioral activation: A simulation study. In UbiComp/ISWC 2017 - Adjunct Proceedings of the 2017 ACM International Joint Conference on Pervasive and Ubiquitous Computing and Proceedings of the 2017 ACM International Symposium on Wearable Computers (pp. 830-843). Association for Computing Machinery. https://doi.org/10.1145/3123024.3125617

\section{General rights}

Copyright and moral rights for the publications made accessible in the public portal are retained by the authors and/or other copyright owners and it is a condition of accessing publications that users recognise and abide by the legal requirements associated with these rights.

- Users may download and print one copy of any publication from the public portal for the purpose of private study or research.

- You may not further distribute the material or use it for any profit-making activity or commercial gain

- You may freely distribute the URL identifying the publication in the public portal 


\section{Supporting Smartphone-based Behavioral Activation: A Simulation Study}

\author{
Jakob E. Bardram, \\ Darius A. Rohani \\ Copenhagen Center for Health Technology \\ Technical University of Denmark \\ Kgs. Lyngby DK-2800, Denmark \\ \{jakba,daroh\}@dtu.dk
}

\author{
Nanna Tuxen, \\ Maria Faurholt-Jepsen, \\ Lars V. Kessing \\ Psychiatric Center Copenhagen \\ University of Copenhagen \\ \{nanna.tuxen, maria.faurholtjepsen, lars.vedel. \\ kessing\}@regionh.dk
}

\section{ABSTRACT}

Behavioral activation has shown to be a simple yet effective therapy for depressive patients. The method relies on extensive collection of patient reported activity data on an hourly basis. We are currently in the process of designing a smartphone-based behavioral activation system for depressive disorders. However, it is an open question to what degree patients would use this approach given the high demand for user input. In order to investigate this question, we collected paper-based behavioral activation forms from 5 patients, covering in total 18 weeks, 115 days, and 1,614 hours of self-reported activity data. In this paper we present an analysis of this data and discuss the implications for the design of a smartphone-based system for behavioral activation.

\section{CCS CONCEPTS}

- Human-centered computing $\rightarrow$ Ubiquitous and mobile computing systems and tools; • Applied computing $\rightarrow$ Consumer health; Psychology; 


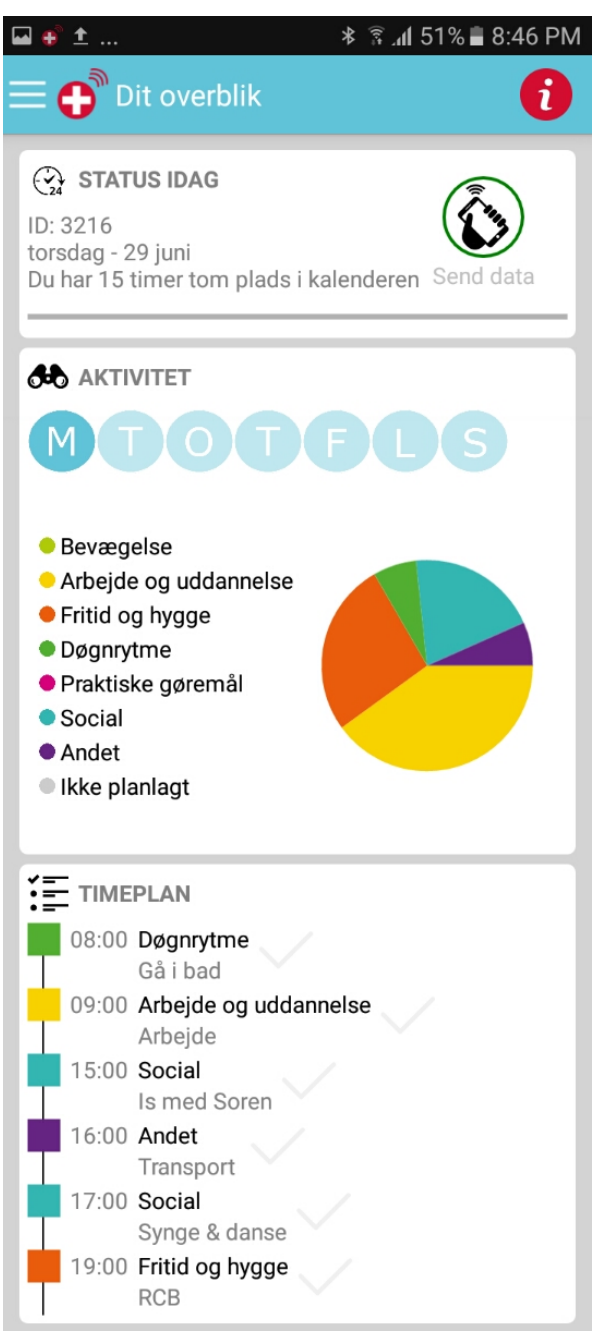

Figure 1: The overview screen of MorıBus.

\section{KEYWORDS}

Mental Health; Depression; Behavioral Activation; Smartphone

\section{ACM Reference format:}

Jakob E. Bardram, Darius A. Rohani and Nanna Tuxen, Maria Faurholt-Jepsen, Lars V. Kessing. 2017. Supporting Smartphone-based Behavioral Activation: A Simulation Study. In Proceedings of UbiComp/ISWC'17 Adjunct, Maui, HI, USA, September 11-15, 2017 (UbiComp/ISWC'17), 14 pages.

https://doi.org/10.1145/3123024.3125617

\section{INTRODUCTION}

Of all registered mental diseases in Europe, unipolar disorder (depression) has the highest prevalence of $6.9 \%$ [13]. This large patient group imposes a significant societal burden with re-admissions, lost productivity, and mortality [11]. The current treatment consists of pharmacotherapy, psychotherapy, or a combination [6]. The most popular method of psychotherapy for depression and many other mental disorders is cognitive behavioral therapy (CBT) [2] due to its short-term consultations and problem solving technique. However, in a large 16-week randomized trail [1] it was found that behavioral activation (BA) alone was as effective as pharmacotherapy and both treatments were significant better than cognitive therapy $(\mathrm{CT})$. The treatment plan for BA starts with the patient reporting his/her activity every hour for several weeks [4]. This is done on a print-out of a week schedule typically between $8 \mathrm{am}$ and $11 \mathrm{pm}$. The activity is provided with a score on 'mastery' (i.e., the level of perceived accomplishment) and 'enjoyment' (i.e., how pleasant the activity was felt) Together with a therapist, the patient then identifies activities that reinforces depressed and healthy behavior [4]. This insight is then used to plan activities of the following week.

Limited clinical personnel together with a growing patient group, have fostered an interest in the use of smartphones to support BA. Smartphones used in behavioral studies and healthcare have been exponentially growing due to its passive sensor data and the ability to prompt users in-the-wild. This yields a powerful combination for studying behavior and for behavioral change interventions [7] However, in a recent review of 117 CBT and BA apps, Huguet et al. [3], finds that there is a "low level of adherence to the core ingredients of the CBT/BA models" and concludes that "the utility of these CBT/BA apps are questionable".

Wahle et al. [10] have developed the most recent system targeting BA for depression. Passive sensors monitoring mobility and physical and social activity are guiding a recommender system to suggest activities. However, this system is based on a pre-made list of activities. This method creates a non-personalized behavioral change intervention that does not learn from the patient's own behavioral traits.

Currently, we are extending this research and are designing a BA system called MorIBus [8]. The aim is to leverage patient generated activity data to develop an automatic recommender of personalized 


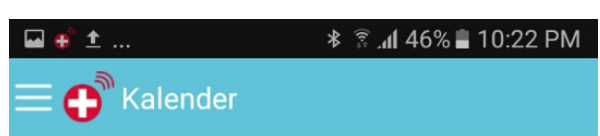

juni 2017

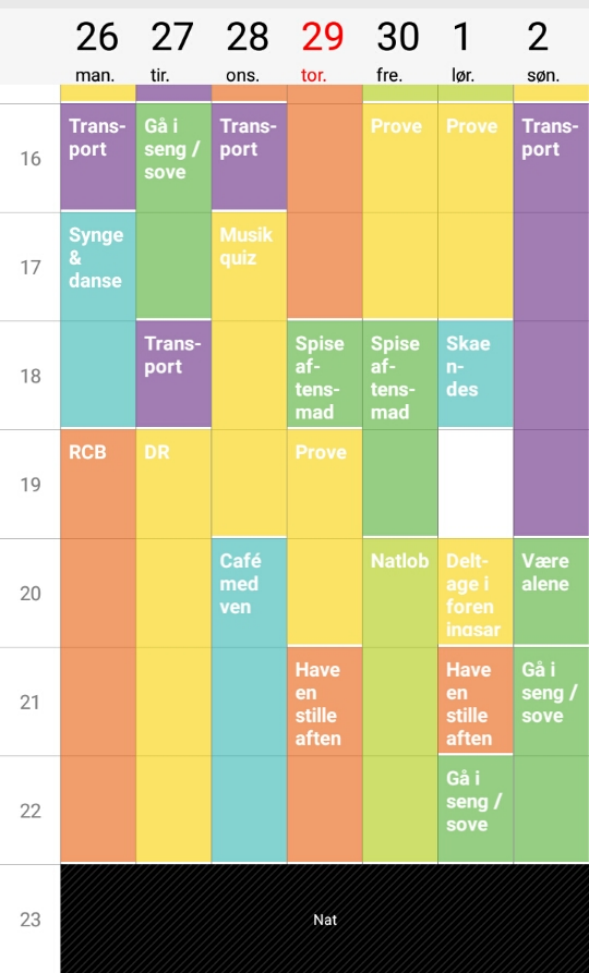

Figure 2: The calendar screen of MoRıBus.

1"Hygge" is a Danish word gradually making its way into the English vocabulary [12]. activities. In order to do this, we first need to sample activities from the patient and analyze which are reinforcers of respectively healthy or depressed behavior. Such a system would have the possibility to improve existing BA methods by assisting inexperienced psychologists to locate possible reinforcers and to provide the patient with a powerful data-driven psycho-educational insight into their own behavior.

However, BA activation requires the patient to do detailed self-reporting of daily activities on a hourly basis. This can be a cumbersome task, whether it is done on paper or on a smartphone. Therefore, in order to investigate the feasibility of the design of MoriBus, we have done a detailed analysis of a set of paper-based activity forms filled in by 5 patients. This analysis contributes to several things:

- It provides an analysis of real-world BA self-reporting.

- It provides a validation test of the design of MoriBus - both in terms of the activity categorization applied and the feasibility of using the smartphone for activity registration.

- It provides insight into how the design of Moribus can be improved.

Even though engagement with paper-based tools does not predict engagement or feasibility of using technology, this study will still provide insight into the details of how patients fill in BA forms and help improve on the design of MoRiBus before further usability testing and field deployment is undertaken.

\section{MORIBUS}

The core design approach of MoriBus is to support the BA method by building a patient-generated database of activities and help the patient to discover reinforcers. MoriBus is designed to be used in a 'blended care intervention' in which a clinicians motivates a patient to use the app for four weeks, whereafter the patient and clinician together reviews the progress. If the patients wants, she or he can continue to use the app.

Every hour between $8 \mathrm{am}$ and $11 \mathrm{pm}$ the patient provides information on the current activity and scores it in terms of 'mastery' and 'enjoyment'. On the basis of Mørch \& Rosenberg [5], we developed six distinct activity categories to cover all types of activities:

Movement - Running, biking, taking a walk, swimming, dancing

Work \& education - Updating CV, doing volunteer work, at the office

Sparetime \& "hygge" - Reading a book, watching TV, shopping, 'hygge'

Daily living - Sleeping, getting up, eating, taking a bath, planning

Practical things - Vacuum cleaning, buying groceries, cooking, gardening

Social - Cup of coffee with a friend, cinema with mom, with guests 

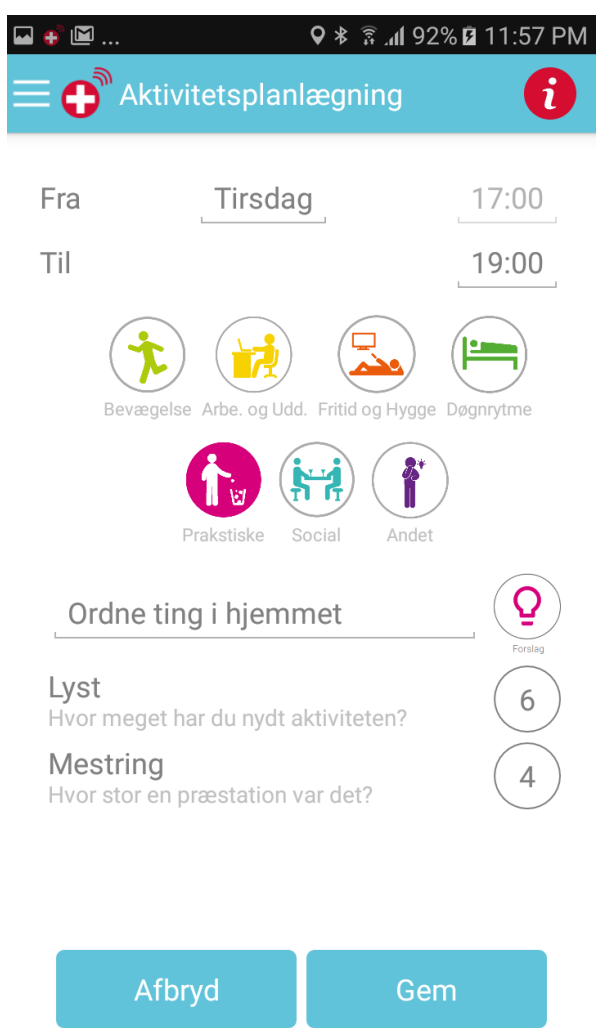

Figure 3: The registration screen of Moribus.
Figure 1 shows the main overview of today's planned activities with a pie chart showing the distribution amongst activity categories. Figure 2 shows a calendar to be used for weekly planning (typically on Sundays). By pressing an empty slot, the patient can plan an activity using the screen shown in Figure 3. The patient selects one of the categories, can choose from a list of standard activities (by pressing the lightbulb icon) or can enter a text for the activity, which then will be saved as part of the list for later use.

Every hour - or when a planned activity ends - Moribus notifies the patient to register the activity using the registration screen (Figure 3). Here the patient enters the details of the activity done, including score the activity in terms of 'mastery' and 'enjoyment' on a $1-7$ scale. At the end of the day, the patient enters a daily mood score on a -3 to 0 scale ( -3 being most depressed).

The application is built on top of SENSUS, an open source system for mobile sensing [14]. The combined data from the activity sampling, daily mood score, and phone sensor data is synchronized from the phones storage to an Amazon S3 storage whenever a wifi connection is available.

\section{ACTIVITY TRACKING}

MoriBus is an example of a system for collecting patient generated data (PGD) on activity. The end goal is to design a fully automated solution for BA therapy, which helps to restructure the patient's activities with recommendations based on own prior activities and activity patterns. However, this requires the patient to self-report activities on an hourly basis, at least for a training and calibration period. A core challenge to this approach is the danger of overloading the user with tedious manual input. In the case of Moribus, prompting patients every hour - particularly patients with a mood disorder - is putting on a large data entry burden on patients. At the workshops done as part of designing MoriBus, patients argued that only "...if the activity sampling can be done with few taps, it will be acceptable" (see [8] for details on the design process).

Our current design tries to meet this design goal. In order to test if this is the case, we did a simulation study of MoriBus by collecting paper-based BA charts from 5 patients.

\section{ANALYSIS OF PAPER-BASED BA FORMS}

\section{Methods}

We asked a psychologist (NT) to collect as many paper-based BA forms from her patients as possible. This resulted in a collection of forms from 5 patients, covering in total 18 weeks of self-reported activity on an hourly basis. Figure 4 shows an example of a filled form using a template with one sheet per week and slots to fill hourly from 8am to midnight. The instruction on top of the form instructs the patient to rate each activity / hour with a 1-10 score, indicating 'how well you feel in any given 


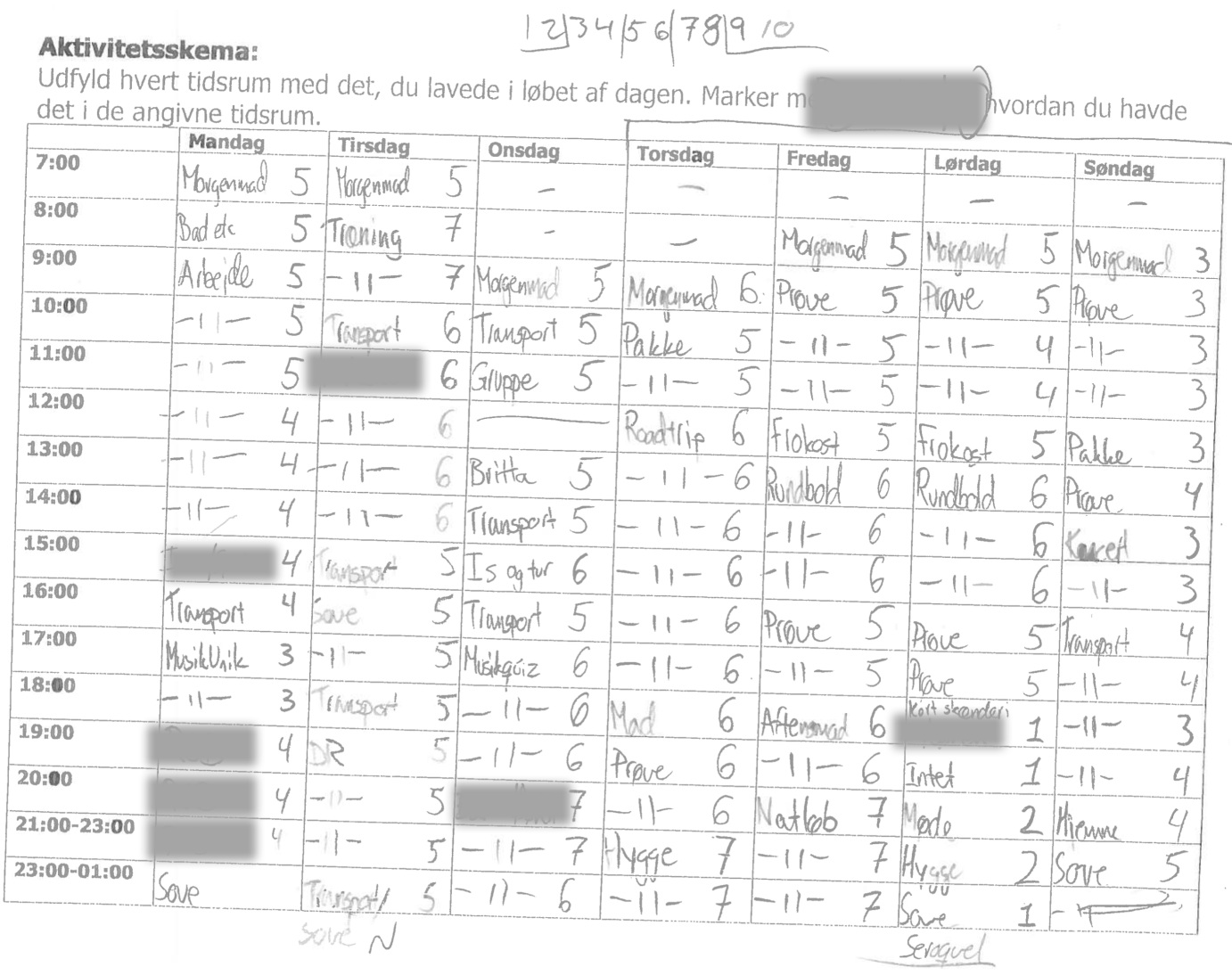

Figure 4: A paper-based BA form from a patient reporting one week of activity every hour on each sheet. In this example, each hours is rated on a general 'feeling' score from 1-10.

timeslot'. We call this score for the 'feeling score'. Each sheet were subject to data extraction and transcription analyzing the following parameters:

- Total numbers of weeks, days, and hours that the patients did self-reporting. 
${ }^{2}$ We have not been able to calculate completion rate for the number of weeks since we have no information on how many weeks/forms each patient was asked to fill out.
P5

P4

33

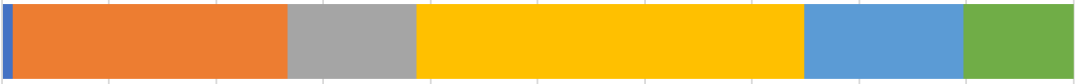

$\mathrm{P} 2$

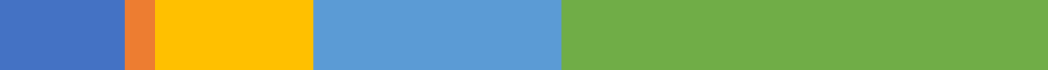

4

Work \& Educ

Sparetime

Daily living

- Prac. things

- Social

- Other

P1

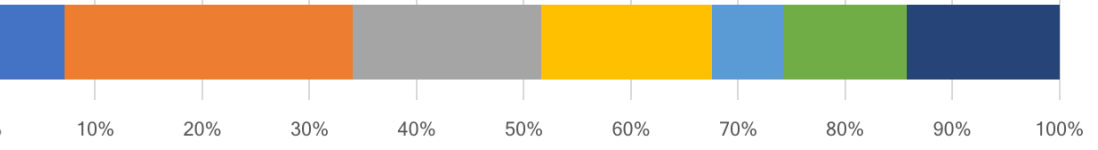

Figure 5: Relative distribution of all activities for all patients.

- Completion rate in self-reporting, calculated as how many of the possible days and hours were actually reported. Full completion was defined as 7 days a week and 16 hours per day (corresponding to the number of slots on the paper form).

- Each hour of self-reported activity with its feeling score (if available) was tagged with one of the predefined activity categories used in MoriBus.

- If the patient has scored the activity, then feeling scores for each activity was transcribed and agregate values (mean and standard deviation) was calculated both on a daily, weekly, and overall scale.

\section{Results}

Table 1 shows an overview of the self-reported data from each patient. In total 5 patients reported 18 weeks, 115 days, and 1,614 hours of self-reported activity data. Overall completion rate (show in parenthesis) is $91 \%$ for the number of self-reported days and $88 \%$ for hours ${ }^{2}$.

Figure 6 (left) shows the distribution of all activities of all patients (1,614 activities in total) categorized into the six Moribus categories. Figure 6 (right) shows the distribution of all activities of P1 (182 in total) into the six MoriBus categories. The piechart resembles the visualization used in MoriBus, as 

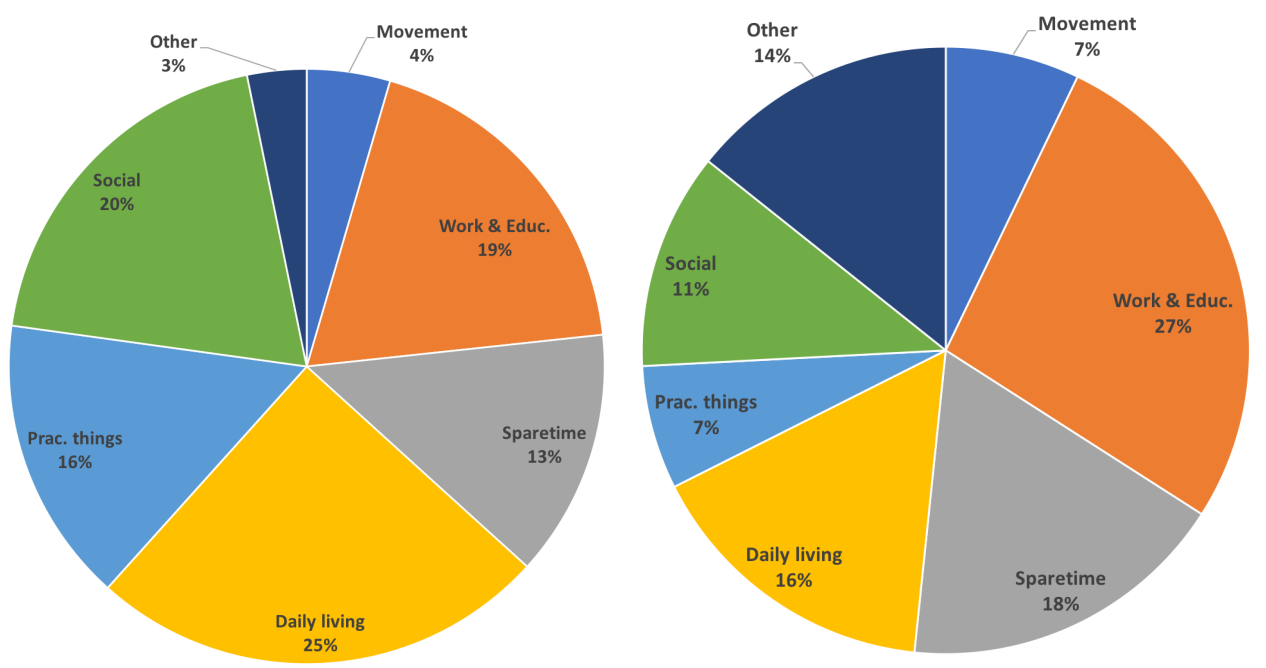

Figure 6: Categorization of activities. Left: All patients. Right: Patient P1. The distribution for all patients is shown in figure 5.

\begin{tabular}{cccc} 
patient & weeks & days & hours \\
\hline P1 & 2 & $12(86 \%)$ & $182(95 \%)$ \\
P2 & 3 & $17(81 \%)$ & $250(92 \%)$ \\
P3 & 4 & $28(100 \%)$ & $398(89 \%)$ \\
P4 & 8 & $51(91 \%)$ & $676(83 \%)$ \\
P5 & 1 & $7(100 \%)$ & $108(96 \%)$ \\
\hline sum & 18 & $115(91 \%)$ & $1,614(88 \%)$ \\
\hline
\end{tabular}

Table 1: Overview of patient and the number of self-reported weeks, days, and hours. Percentage in parenthesis is completion rate. shown in Figure 1, except that Figure 6 shown the distribution for all 12 days, whereas the pie chart in Moribus shows the distribution for one day. Figure 5 shows the relative distribution of activities for all patients.

Regarding patient self-rated feeling scores of each activity, we found that two patients (P1 and P2) used a 1 - 10 scale for indicating general feeling; P3 used a $1-5$ scale, and P4 and P5 did not score their activities. It should be noted that P4 and P5 were never asked to do the scoring by the therapist. Figure 7 shows a box plot of the feeling score according to each activity category (average and standard deviation) for patient P1, P2, and P3.

Figure 8 shows the average feeling score per day for $\mathrm{P} 3$ over the 4 -week period. This graph illustrates the development of feelings over the week and over the 4-week period, while also showing feeling scores per week day. P3 was the patient with most self-reported activity data, which also included feeling scores. 

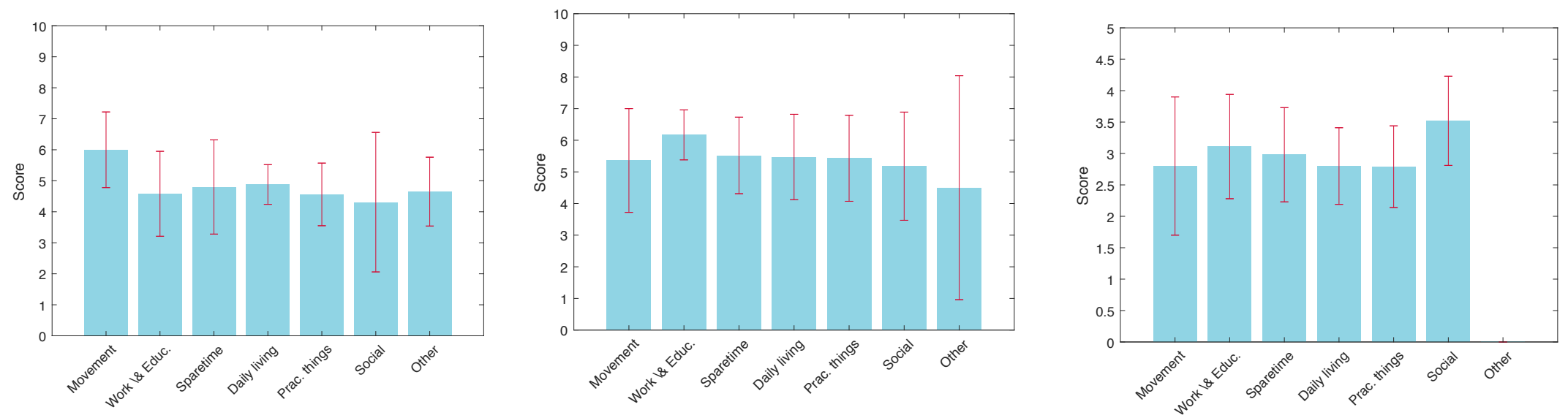

Figure 7: Feeling scores (average and standard deviation) for patient P1, P2, and P3.

\section{DISCUSSION}

As stated in the Introduction, the purpose of this study was to provide an analysis of real-world BA self-reporting, and use this as a validation test of the activity categorization applied in MoRIBUs, which then can provide an insight into how the design of MoriBus can be improved.

\section{Completion Rate}

A primary research question in this study was; to what degree would patients fill in BA forms on a weekly basis with activity registration on an hourly basis. This is a core design assumption of MoriBus, and the usefulness of the system as well as the validity of the data it collects, rely on the degree to which patients would do this. From Table 1 we see that the overall completion rate is high; all are in the 80 s, and 3 out of 5 patients have a completion rate in terms of reported hours above $90 \%$. Overall completion rate in terms of hours is $88 \%$ from reporting 1,1614 hours in total. These figures indicate that when a motivated patient is asked to fill in BA forms, he or she does this with a very high degree of completion. This provides positive evidence for the feasibility of moving this type of BA forms and intervention to a smartphone platform. 


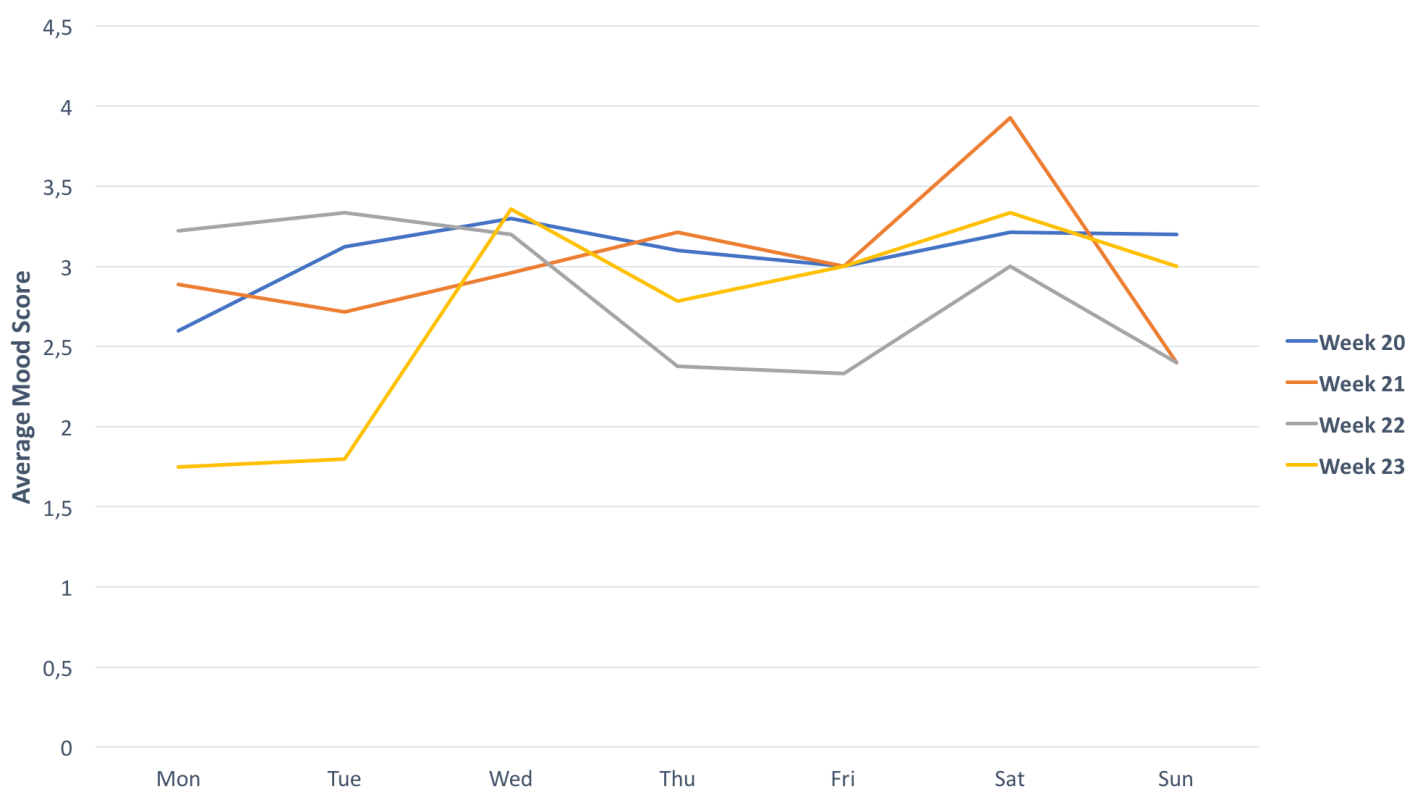

Figure 8: Average feeling scores per day over the 4-week period for P3.

\section{Activity Categories}

A second research question we asked was; would the six activity categories suffice to map realworld activities? Looking at Figure 6 (left) only 3\% (52 activities) fall in the 'other' category, meaning that they could not be categorized into the predefined six MoRiBus categories. Hence, the vast majority of realworld activities could be categorized. However, the transcription revealed a set of typical activities to select from the suggestion page of MoRIBus were missing. These were:

- Transport (e.g., driving, biking, taking the train)

- Relationship (e.g., dating, having dinner w. boyfriend, sleeping over)

- Illness (e.g., being ill, treatment for depression, psychotherapy)

- Children (e.g., playing with the kids, bedtime for kids, bathing kids)

- Social media (e.g., facebook, instagram)

- Religion (e.g., ceremony, baptise) 
The transcript also revealed that the order of the predefined suggestions in the MoriBus app were not optimal - some of the most frequently used suggestions were long down the list. Moreover, the app seems to suffer from a 'cold start' problem, i.e., the calendar and the overview display are completely empty and there are no historical data or graphs to gain any insight from. It is therefore difficult for patients to get started. Based on this analysis, the activity suggestion in MoriBus is currently being optimized and some pre-defined and pre-planned activities are available for the patient to get started. For example, a pre-planned activity called 'Planning my week' is scheduled for Sunday afternoon at $4 \mathrm{pm}$.

\section{Activity Profiles}

In order to understand how different patients are coping with their depression and to investigate if there were any common patterns, we analyzed the categorization of activities for each patient (Figure 5). The analysis was done by identifying which types of activities were most dominant both quantitatively (in terms of hours spend on this type of activity) as well as qualitatively (in terms of which types of activities seemed to improve the feeling score, if available). Through this analysis, different 'activity profiles' was identified:

Maintaining a professional life - Some patients focus on maintaining their professional life by continuing to work and/or attending school. A normal work week in Denmark is 37 hours, which means that if a patient is able to work she or he would spend 33\% (37 out of 112 hours) of her or his time working. None of the patients are at $33 \%$ but P3 is close (27\%).

Maintaining everyday life - Some patients focus on activities of daily living at home such as preparing proper meals, eating, resting and sleeping. All patients clearly need to do this, but for some it takes up a larger proportion of the week than others. For example, P1 and P3 spend 25\% and $36 \%$ of their time doing daily activities, staying at home.

Maintaining a social life - Some patients spend time and effort on relating to others and spend a proportionally large amount of time with other people. P4 and especially P5 fits this activity profile.

Maintaining physical activity - Physical activity such as going for a walk, bicycling, jogging, swimming, etc. may improve recovery from depression [9]. From the study, we see that P5 seems to very active, spending $14 \%$ of his or her time on activities in the 'movement' category. The other patients seems to be less active, ranging from 1-7\%. However, it should be noted that one hour of physical activity - like jogging - does not compare equally to one our or 'hygge', like watching a movie. The Danish health authorities recommend 30 minutes of physical activity every day, which equals 4 hours per week, which equals $4 \%$. Hence, all patients except P3 actually complies to this recommendation. 


\section{Feeling Scores}

When analyzing the 'feeling scores' this study was less conclusive. There was a lack of standardization in the scoring methodology; P1 and P2 used a $1-10$ scale, P3 used a $1-5$ scale, and P4 and P5 did not score their activities. Also, the instruction stating that the patient should 'rate how you feel' is a very vague and imprecise instruction, which was subject to different interpretations by the patients. For example, P2 used the scale continuously, meaning 1 is worst and 10 is best. But P1 divided the scale into 5 sub-scales [ $1-2][3-4][5-6][7-8][9-10]$ and defined the lowest interval as 'hopeless' and the highest scale as 'unhealthy / manic'. Hence, even though P1 and P2 used the same scale, their scores are incomparable.

Analyzing the feeling scores according to activity categories as shown in Figure 7 shows no clear patterns; scores in all categories seems to be similar both in terms of average score and median. P2 has a large standard deviation for activities in the 'other' category, but this reflects the fact that this contains both activities on 'church visit' (which is rated very low) as well as 'psychotherapy' (which is rated very high). The only finding seems to be that P1 feels better when being physically active ('movement') as compared to other kinds of activities; similarly with P3 on social activities.

However, when doing a more detailed analysis of the feeling score over the course of a week for P3 as shown in Figure 8, we do find an interesting pattern. It seems like the general feeling increased on Saturdays, declined on Sunday and then increased again on Wednesdays (mid-week). This observation could be relevant for P3, since it might help her or him to better prepare for Sundays and maybe plan 'nice' activities beforehand (a key approach in BA).

\section{FEASIBILITY TEST OF MORIBUS}

As an additional verification step of the MoriBus system, we did a simulation based on these realworld data. Specifically, we wanted to investigate the following questions:

(1) Will the user be able to enter his or her realworld data, i.e. does MoRiBus support the activity registration from the paper-based forms?

(2) How much time would a user spend on planning activities - both for a day and for a whole week?

(3) How much time would a user spend on registrering 'mastery' and 'enjoyment' for activities both planned and non-planned activities?

In order to investigate these three questions, one author (JEB) took the data from one patient - P1 - and entered it into MoriBus to see how this worked. Specifically, we entered the data for one week from the paper-based form shown in Figure 4. The results are shown in Table 2.

Overall, there was no problems in entering the form into Moribus. In fact, the overview screen and calendar shown in Figure 1 and 2 show data taken from the paper form shown in Figure 4 . When 


\begin{tabular}{rcc} 
& time & \# new act. \\
\hline Planning a day & $03: 07$ & 3 \\
Planning a week & $19: 34$ & 16 \\
Registration of a planned day & $01: 18$ & 0 \\
Registration of an unplanned day & $02: 15$ & 0 \\
\hline
\end{tabular}

Table 2: Timing of entering and registration of activities in Moribus - for a day and for a week. entering the data for one week, 16 new activities in total had to be manually created (i.e., they were not part of the standard list). This corresponds to $9 \%$ of the total amount of activities registred by P1.

As for the timing of using MoRiBus for activity planning and registration, we see that it took a little over 3 minutes to plan one day and ca. 20 minutes to plan a 7-day week. This includes the entry of 3 and 16 new activities for a day and week, respectively. Once these activities has been entered into Moribus, future planning will be slightly faster since these activities are now available.

The registration of planned activities for a day was fast - less than 2 minutes. Moreover, we see that planing and registration of a day was faster ( 2 minutes) compared to just planning. This is because in the latter case (i.e. the first round), 3 new activities had to be entered into the system. Hence, once activities are entered and available, then registration of unplanned but similar activities becomes faster.

We are well aware that comparing the time and effort of a designers of MoRiBus doing a desk test with a patient less familiar with the app in a realworld setting might be less valid. We do expect however, that once familiar with Moribus, patients will be able to plan and register activities within a similar timeframe. And we consider this a reasonable time usage; spending a couple of minutes each day to plan or register activities are definitely acceptable. Spending 20 minutes to plan a whole week is below the one hour that a therapist recommend a patient to spend each Sunday - even though one hour clearly also should include time for contemplating about activities for the upcoming week, and not only interacting with the smartphone.

\section{CONCLUSION}

Behavioral activation (BA) has proven to be a simple yet efficient therapy approach for depression that encourage patients to do detailed reporting of activities on an hourly basis. To support BA on a smartphone we have developed MoriBus. In order to validate the feasibility and design of the system, we did a simulation of the use of it. The simulation was done by collecting paper-based BA forms from 5 patients, covering in total 18 weeks, 115 days, and 1,614 hours of self-reported activity data. A thorough analysis of this bulk of data revealed that the completion rate of all patient was very high, which indicates that this kind of activity registration is feasible to ask patients to perform. Moreover, the data showed that the categories used in Moribus were sufficient to cover all 1,614 registered hours of activity. Only a few generic activities needed to be added to the standard list in Moribus. The data analysis also revealed a set of prototypical 'activity profiles', including patients focusing their BA activities on professional, everyday, social, and physical activities.

Analyzing the 'feeling' scores of the patients, the study was, however, less conclusive. It became apparent that patients use the scales very differently (if at all), and that there were no consistent patterns in terms of relationship between the feeling score and the types of activities done. The analysis of four weeks of feeling scores for one patient, however, revealed that there seemed to be a 
weekly cyklus in her or his scores; Sundays are generally low, whereas Saturdays are the 'peak' of the week. This kind of visualization of patterns might help the patient get an insight the cyklus of her or his feelings and plan accordingly - a methods which is the cornerstone of BA.

Based on this analysis, MoriBus is currently being optimized and made ready for general deployment and clinical testing.

${ }^{3} \mathrm{http} / /$ www.cachet.dk/research/projects/radmis

${ }^{4} \mathrm{http}: / /$ www.cachet.dk/

\section{ACKNOWLEDGMENTS}

This research is done as part of the RADMIS project ${ }^{3}$ funded by the Innovation Fund Denmark and the Copenhagen Center for Health Technology $(\mathrm{CACHET})^{4}$.

\section{REFERENCES}

[1] Sona Dimidjian, Steven D Hollon, Keith S Dobson, Karen B Schmaling, Robert J Kohlenberg, Michael E Addis, Robert Gallop, Joseph B McGlinchey, David K Markley, Jackie K Gollan, David C Atkins, David L Dunner, and Neil S Jacobson. 2006. Randomized trial of behavioral activation, cognitive therapy, and antidepressant medication in the acute treatment of adults with major depression. Journal of consulting and clinical psychology 74, 4 (2006), 658-70. https://doi.org/10.1037/ 0022-006X.74.4.658

[2] Stefan G Hofmann, Anu Asnaani, Imke JJ Vonk, Alice T Sawyer, and Angela Fang. 2012. The efficacy of cognitive behavioral therapy: A review of meta-analyses. Cognitive therapy and research 36, 5 (2012), 427-440.

[3] Anna Huguet, Sanjay Rao, Patrick J. McGrath, Lori Wozney, Mike Wheaton, Jill Conrod, and Sharlene Rozario. 2016. A systematic review of cognitive behavioral therapy and behavioral activation apps for depression. PLoS ONE 11, 5 (2016), 1-19. https://doi.org/10.1371/journal.pone.0154248

[4] C.W. Lejuez, Derek R. Hopko, James P. LePage, Sandra D. Hopko, and Daniel W. McNeil. 2001. A brief behavioral activation treatment for depression. Cognitive and Behavioral Practice 8, 2 (2001), 164-175. https://doi.org/10.1016/S1077-7229(01) 80022-5

[5] Merete M Mørch and Nicole K Rosenberg. 2005. Kognitiv terapi: modeller og metoder. Gyldendal A/S.

[6] The National Institute of Mental Health. 2016. Depression - Treatment and Therapies. (2016). https://www.nimh.nih.gov/ health/topics/depression/index.shtml\#part_145399 [Online; accessed 16-March-2017].

[7] Veljko Pejovic, Neal Lathia, Cecilia Mascolo, and Mirco Musolesi. 2015. Mobile-Based Experience Sampling for Behaviour Research. arXiv preprint (2015), 1-20. https://doi.org/10.1007/978-3-319-31413-6_8 arXiv:1508.03725

[8] Darius Rohani, Nanna Tuxen, Lars V Kessing, and Jakob E Bardram. 2017. Designing for Hourly Activity Sampling in Behavioral Activation. In Proceedings of the 2017 ACM International Joint Conference on Pervasive and Ubiquitous Computing. Adjunct. ACM, 1162-1170.

[9] Megan Teychenne, Kylie Ball, and Jo Salmon. 2008. Physical activity and likelihood of depression in adults: A review. Preventive Medicine 46, 5 (2008), 397 - 411. https://doi.org/10.1016/j.ypmed.2008.01.009

[10] Fabian Wahle, Tobias Kowatsch, Elgar Fleisch, Michael Rufer, and Steffi Weidt. 2016. Mobile Sensing and Support for People With Depression: A Pilot Trial in the Wild. JMIR mHealth and uHealth 4, 3 (2016), e111. https://doi.org/10.2196/mhealth.5960

[11] Philip S Wang, Gregory Simon, and Ronald C Kessler. 2003. The economic burden of depression and the cost-effectiveness of treatment. International journal of methods in psychiatric research 12, 1 (2003), 22-33.

[12] Meik Wiking. 2016. The Little Book of Hygge: Danish Secrets to Happy Living. Penguin Life

[13] Hans-Ulrich Wittchen and Frank Jacobi. 2005. Size and burden of mental disorders in Europe - a critical review and appraisal of 27 studies. European neuropsychopharmacology 15, 4 (2005), 357-376. 
[14] Haoyi Xiong, Yu Huang, Laura E. Barnes, and Matthew S. Gerber. 2016. Sensus: A Cross-platform, General-purpose System for Mobile Crowdsensing in Human-subject Studies. Proceedings of the 2016 ACM Int. Joint Conf. on Pervasive and Ubiquitous Comp. (2016), 415-426. https://doi.org/10.1145/2971648.2971711 